\title{
An Intermediate formed in Sulphonation of Iodobenzene
}

\author{
N. H ES SE L B J ER G C H R IS T EN SEN
}

Chemistry Department, Research Establishment Ris $\phi$, Denmark

\begin{abstract}
An unstable coloured complex, invariably accompanying the sulphonation of iodobenzene, has been investigated spectrophotometrically. The spectrum shows maximum absorption at $400-380 \mathrm{~m} \mu$, in nitromethane, 1,2-dichloroethane as well as in fuming sulphuric acid. An investigation has been carried out of the reaction at room temperature with nitromethane as solvent, using a fixed wave-length of $380 \mathrm{~m} \mu$.

The kinetics are consistent with the hypothesis that the colouring compound is a 2:1 complex of iodobenzene and sulphur trioxide. It is discussed whether this complex may represent an intermediate stage in the sulphonation reaction.

By sulphonation of chloro- and bromobenzene with sulphur trioxide in dichloroethane, similar absorption phenomena are detected at $290-300 \mathrm{~m} \mu$.
\end{abstract}

$\mathbf{I}_{\mathrm{r}}^{\mathrm{t}}$ t has now been widely accepted that the aromatic electrophilic substitution reactions proceed by way of intermediates ( $c f$. Ingold ${ }^{1}$ and Brown and Nelson ${ }^{2}$ ). The sulphonation reaction does not constitute an exception in this respect; the evidence for an intermediate having been claimed on account of kinetic data. Hinshelwood et $a l \cdot{ }^{3-5}$ observed that sulphonation with sulphur trioxide in nitrobenzene was dependent upon the sulphur trioxide concentration squared, and although other conclusions were drawn by the observers, the observation was later interpreted by Ingold, ${ }^{1}$ Brown and Nelson ${ }^{2}$ and Gilbert ${ }^{6}$ in favour of a two-stage mechanism involving an intermediate designed as a complex between sulphur trioxide and the aromatic substrate. Melander ${ }^{7}$ and Berglund-Larsson ${ }^{8}$ stated the existence of a low, but significant, kinetic isotopic effect when sulphonating mixtures of bromo-benzene and bromobenzene-4- ${ }^{3} \mathrm{H}$, and they explained this by assuming a two-stage mechanism with the second stage partly rate-determining. Brand et al. ${ }^{9}$ obtained support for a similar mechanism, using conventional kinetics, as well as kinetic isotopic effects of deuterium in various aromatic substrates or in the sulphonating medium. Gold and Satchell ${ }^{10}$ came to similar conclusions when studying sulphonation of benzene in sulphuric acid. Davenport and Walsh ${ }^{11}$ proposed a similar mechanism to account for kinetics observed in a study of the sulphonation of anisole with sulphur trioxide in dioxane. 
By preparing $p$-iodobenzene sulphonic anhydride, $\left(\mathrm{IC}_{6} \mathrm{H}_{4} \mathrm{SO}_{2}\right)_{2} \mathrm{O}$, by mixing iodobenzene and sulphur trioxide in nitromethane solution ${ }^{12}$ it has invariably been observed in this laboratory that the mixture instantaneously takes on a yellow-brown colour, which thereafter disappears in the course of $20-200$ sec.

It was found of interest to undertake a more detailed investigation of the phenomenon, in view of the possibility that the colour was due to an intermediate. The reaction was therefore studied spectrophotometrically in nitromethane solution at room temperature, and the rate data have been analysed. In addition some rate measurements on product formation in the system were made.

The coloured compound is also formed when dichloroethane or oleum is used as solvent for the reaction. By the sulphonation of chlorobenzene and bromobenzene no absorption in the visible range is observed, but a temporary absorption in the neighbourhood of $300 \mathrm{~m} \mu$ can be detected.

\section{EXPERIMENTAL}

Choice of experimental conditions. Nitromethane was chosen as the solvent-medium for the sulphonation process, since the nature and the distribution of the reaction products formed by sulphonation of iodobenzene in this medium are well known.12

It has been mentioned in the literature that a solution of sulphur trioxide in nitromethane may undergo a violent reaction with evolution of gases. ${ }^{13}$ In spite of more than a hundred preparations of such solutions in this laboratory, reactions of this sort were never observed, unless water, deliberately or by accident, was added to the solution. However, nitromethane is not completely inert to sulphur trioxide. A $1 \mathrm{M}$ solution stored at room temperature decomposes with a half-life of about $20 \mathrm{~h}$, as estimated from its capacity of forming iodobenzene sulphonic anhydride. A solution of sulphur trioxide in nitromethane should therefore be prepared immediately before use, or be stored as a frozen solid, e.g. by cooling in dry ice.

The precipitation of the iodobenzene sulphonic anhydride proved disadvantageous in the present investigation, and several runs had to be discarded on this account. How ever, with initial $\left[\mathrm{SO}_{3}\right] /\left[\mathrm{C}_{6} \mathrm{H}_{5} \mathrm{I}\right]$ ratios less than 1 , precipitation was, as a rule, avoided, provided the cuvettes had been thoroughly cleansed.

Dichloroethane was used as solvent in some supplementary experiments. This compound has previously been applied as solvent in the study of sulphonation and has proved very stable towards sulphur trioxide. ${ }^{13-14}$ With this solvent precipitation rarely occurred.

Finally, it was attempted to use fuming sulphuric acid as solvent and reagent. The high viscosity and partial heterogeneity of the mixture of iodobenzene and sulphuric acid complicate rate experiments.

Materials. Nitromethane and 1,2-dichloroethane of purest quality from Fluka were dried over $\mathrm{CaCl}_{2}$ and distilled in thoroughly dried equipment.

The purity of the nitromethane is important for a high yield of sulphonic anhydride and the quality of the solvent is also of importance for the phenomenon studied in the present investigation. When a nitromethane of technical grade is applied, the shortliving brownish colour may be more or less obscured by a more stable red colour.

Weighed samples of sulphur trioxide, $0.2-1.0 \mathrm{~g}$, were prepared by drawing the vapour from $50 \%$ oleum into a U-tube, cooled in dry ice, by means of a stream of dry nitrogen.

Solutions of sulphur trioxide were prepared by adding measured volumes of the solvent. A little gelatinous material was always left undissolved when applying dichloroethane, and this material was discarded before further operations. The molarity was controlled by titration.

In some kinetic runs on the colour reaction an amount of $100 \%$ sulphuric acid was added to the sulphonation mixture. The acid was prepared by mixing $98 \%$ sulphuric acid with sulphur trioxide until the total content of $\mathrm{SO}_{3}$ in a weighed sample, as determined by titration, was $81.5 \pm 0.2 \%(\mathrm{w} / \mathrm{w})$. 
The chloro-, bromo- and iodobenzene were purest qualities from Merck and British Drug Houses. They were first washed with an alkaline solution, then with acid, finally with water. After drying over $\mathrm{Na}_{2} \mathrm{SO}_{4}$ the samples were fractionated at reduced pressure, the middle fractions being used. The chlorobenzene had b.p. $58.0^{\circ}$ at $62 \mathrm{~mm}$, bromobenzene $77.2^{\circ}$ at $60 \mathrm{~mm}$ and iodobenzene $73^{\circ}$ at $16 \mathrm{~mm} \mathrm{Hg}$.

A sample of the iodobenzene was analysed by gasphase chromatography. It was concluded that components with b.p. outside the range $189^{\circ} \pm 5^{\circ}$ did not constitute more than $0.2 \%$ of the sample. The technique applied did not effectively exclude such likely contaminants as aniline, b.p. $184^{\circ}$, and perhaps phenol, b.p. $182^{\circ}$. However, it is easy to prove that neither causes colour phenomena by sulphonation.

Colour spectra. Absorbance was recorded on a Perkin-Elmer Spectracord Model 4000. Due to absorption of the solvent, work with nitromethane had to be confined to the visible and near-UV range down to $370 \mathrm{~m} \mu$. With dichloroethane the range could be extended down to $230 \mathrm{~m} \mu$. One centimeter quartz cuvettes were used. Spectra of the components were measured against the pure solvents.

As to the colour phenomenon itself, its short lifetime prevents automatic recording of its spectrum. This spectrum was therefore obtained by measuring identical mixtures at different fixed wave-lengths against the pure solvent, and by plotting the maximum absorbance (optical density) against the wave length.

Colour rate experiments. The colour reaction was recorded automatically by measuring the absorbance of a sample at a fixed wave length $(380 \mathrm{~m} \mu)$ against the solvent as a blank. The recording drum was driven at a constant speed of $1.94 \mathrm{~mm} / \mathrm{sec}$. Most runs were performed at room temperature.

After having placed a $1 \mathrm{~cm}$ quartz cuvette containing $2.0 \mathrm{ml}$ of a sulphur trioxide solution $(0.2-1.0 \mathrm{M})$ in the apparatus and started recording, a known volume of iodobenzene $(100-1000 \mu \mathrm{l})$ was quickly added to the solution from a micropipette. Inspection showed this operation to give a rapidly mixed homogeneous solution.

In experiments in which sulphuric acid had been added, it proved more difficult to obtain a homogeneous solution, when iodobenzene was added, and increasingly so, the higher the concentration of the sulphuric acid. After a run, the cuvette was always inspected for precipitation or heterogeneity, and in case such had occurred, the run was discarded.

The initial concentration of the components were calculated from the molar concentration of the sulphur trioxide, and the volumes, assuming ideal conditions of mixing.

While the kinetic data of short-time experiments were fairly consistent, this was not the case with data obtained on different days using different sulphur trioxide stock solution. The lack of consistency is probably due to small, unknown amounts of water and to lack of temperature control in the cuvette chamber, temperatures between $24^{\circ}$ and $30^{\circ}$ having been measured.

In some experiments the temperature in the solution was measured with a thermocouple, and no heat of reaction could be detected. However, it stands to reason that in case of unproperly dried iodobenzene, the water will cause a sulphonation loss as well as a rise in temperature.

In a few experiments measurements at lower temperatures were attempted by cooling the cuvettes with sulphur trioxide solution prior to insertion in the cuvette chamber; the temperature being recorded during the optical measurements. The method sufficed to demonstrate an effect of the temperature on the colour formation rate.

Product rate experiments. Conventional rate experiments on the sulphonation were carried out on mixtures containing sulphur trioxide and iodobenzene in concentrations, and preferably at temperatures, where the rate was not too fast; nitromethane being used as solvent. Most runs were performed at $0^{\circ}$, a few at $20^{\circ}$ and $40^{\circ}$. The mixing was done as in the colour rate experiments, complete mixing in this case being ensured by a gentle flow of nitrogen. At the required time the entire reaction mixture was rapidly transferred to a flask containing $25 \mathrm{ml}$ of water kept in vigorous rotation by a magnetic stirrer. The transfer operation was carried out in a fraction of a second by means of a suction device. The time lapse between mixing of the components and the interruption could be reduced to about one second. The error in the determination of the starting point was about one second, and this error contributes considerably to the error in the initial rate of product formation.

Acta Chem. Scand. 17 (1963) No. 8 
The water samples were heated for $5 \mathrm{~min}$, possible pyrosulphonic acid, $\mathrm{RSO}_{3} \mathrm{SO}_{3} \mathrm{H}$, and sulphonic anhydride, $\left(\mathrm{RSO}_{2}\right)_{2} \mathrm{O}$, thereby being hydrolysed to sulphonic acid, $\mathrm{RSO}_{3} \mathrm{H}$. It has been found that desulphonation of the $p$-iodobenzene sulphonic acid, $\mathrm{H}_{2} \mathrm{O}+$ $\mathrm{RSO}_{3} \mathrm{H} \rightarrow \mathrm{RH}+\mathrm{H}_{2} \mathrm{SO}_{4}$, does not take place to a measurable extent at these conditions. After cooling, a major part of the nitromethane and most of the unreacted iodobenzene was removed by extraction with $15 \mathrm{ml}$ of chloroform. The aqueous layer was diluted to a known volume in $7 \% \mathrm{HCl}$, and the content of $p$-iodobenzene sulphonic acid was determined spectrophotometrically using an absorption peak at $241 \mathrm{~m} \mu$, the molar extinction coefficient being 15600 absorbance units. Blanks and control samples have shown that neither nitromethane nor iodobenzene will cause significant errors, when the above procedure is followed. The concentration of reaction products in the reaction mixture has been calculated from this analysis and from the total volume of the reaction mixture, and is referred to as $\left[\mathrm{RSO}_{3} \mathrm{H}\right]$. The concentration of reaction product after one hour has been taken as the concentration at infinite time, $\left[\mathrm{RSO}_{3} \mathrm{H}\right]_{\infty}$, a few runs having shown the concentrations after $15 \mathrm{~min}, 1 \mathrm{~h}$ and $20 \mathrm{~h}$ to be identical. $\left[\mathrm{RSO}_{3} \mathrm{H}\right]_{\infty}$ has been taken as the initial sulphur trioxide concentration, $\left[\mathrm{SO}_{3}\right]_{0}$.

\section{RESULTS}

Spectra of colours. The indirectly obtained spectrum of the mixture of iodobenzene and sulphur trioxide in nitromethane, $c f$. above, shows maximum absorption at $380-400 \mathrm{~m} \mu, c f$. Fig. 1, and very similar spectra have been ob-

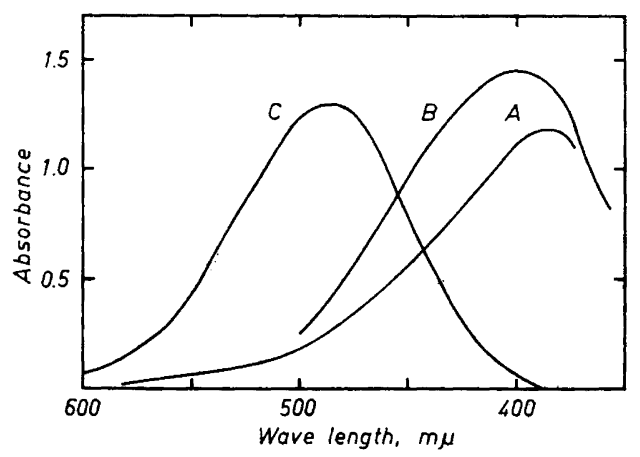

Fig. 1. Visible and near-UV spectra of coloured complex in nitromethane (A) and in dichloroethane $(B) . C$ is spectrum of iodine in nitromethane solution. $A:\left[\mathrm{C}_{6} \mathrm{H}_{5} \mathrm{I}\right]_{0}=1.0$, $\left[\mathrm{SO}_{3}\right]_{0}=0.30 \mathrm{M}$, nitromethane. $\mathrm{B}:\left[\mathrm{C}_{6} \mathrm{H}_{5} \mathrm{I}\right]_{0}=0.08,\left[\mathrm{SO}_{3}\right]_{0}=0.10 \mathrm{M}$, dichloroethane. $\mathrm{C}:\left[\mathrm{I}_{2}\right]=0.0017 \mathrm{M}$, nitromethane. $24-30^{\circ} .1 \mathrm{~cm}$ quartz cuvettes.

tained with dichloroethane and fuming sulphuric acid. It should be pointed out that it was not possible to derive the specific extinction coefficient of the colouring compound.

The sulphonation of chlorobenzene and bromobenzene with sulphur trioxide in dichloroethane does not show a temporary absorption in the visible range, but at wavelengths in the vicinity of $300 \mathrm{~m} \mu$ absorption phenomena of a very similar nature can be detected. Unfortunately the absorption of the reaction products prevents investigation below $290 \mathrm{~m} \mu$.

The influence of iodine. Iodine in nitromethane, with or without reagents or products, absorbs light in the visible range, causing a colour superficially 
like the colour accompanying the sulphonation of iodobenzene. The spectrum has a maximum at $487 \mathrm{~m} \mu$, cf. Fig. 1. Spectra of the sulphonation mixtures taken after the reaction normally show a weak maximum at this wave-length, indicating that some iodine has been formed by a side reaction. The quantity of this iodine does, however, not exceed 1 mole per 100 moles of the total reaction products.

In some rate experiments iodine had been added to the sulphur trioxide solution before addition of the iodobenzene. In a run with initial molar concentrations $\left[\mathrm{C}_{6} \mathrm{H}_{5} \mathrm{I}\right]_{0}=0.82,\left[\mathrm{SO}_{3}\right]_{0}=0.27$ and $\left[\mathrm{I}_{2}\right]_{0}=0.0017$, the visible spectra before and after reaction were recorded and compared. The reaction had had no influence on the iodine spectrum.

Finally it has been observed, cf. below, that iodine at this concentration has little influence on the rate of decomposition of the coloured compound.

On the above evidence it was concluded that the colour in question is not due to free iodine.

Simple colour rate experiments. A typical curve, as recorded by the Spectra-

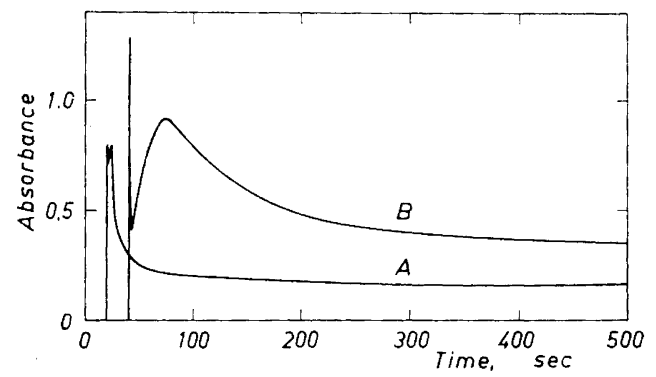

Fig. 2. Spectrophotometric record of absorbance at $380 \mathrm{~m} \mu$ during the sulphonation of iodobenzene, $A$ without and $\mathrm{B}$, with sulphuric acid. $\mathrm{A}:\left[\mathrm{H}_{2} \mathrm{SO}_{4}\right]_{0}=0,\left[\mathrm{SO}_{3}\right]_{0}=0.33$, $\left[\mathrm{C}_{6} \mathrm{H}_{5} \mathrm{I}\right]_{0}=0.82$ M. B: $\left[\mathrm{H}_{2} \mathrm{SO}_{4}\right]_{0}=0.75,\left[\mathrm{SO}_{3}\right]_{0}=0.10,\left[\mathrm{C}_{6} \mathrm{H}_{5} \mathrm{I}\right]_{0}=1.81 \mathrm{M} .24-30^{\circ}$, nitromethane solution. $1 \mathrm{~cm}$ quartz cuvettes.

cord, is shown in Fig. 2 A. At room temperature the colour is formed at a rate of the order of $0.5-2.5 \mathrm{abs}$. units/sec or more, which is comparable to the maximum speed of the recorder, 2.5 abs. units/sec. The formation rate is therefore too fast for quantitative considerations.

As a rule two maxima appear, the first being due to overshooting of the recorder, while the second is a true maximum, normally occurring $1-5$ sec after the moment of mixing, $c f$. also Table 1. The optical density thereafter starts decreasing, following a hyperbolic curve. After 100-400 sec the optical density is constant, but not zero, since the reference sample is the pure solvent and the excess iodobenzene effects some absorption at $380 \mathrm{~m} \mu$. The horizontal branch of the colour curve is therefore used as zero line.

Linearity is obtained when the reciprocal of the optical density is plotted against time, and the reaction is therefore of second order; cf. Fig. 3 . If $A$ 
Table 1. Data from spectrophotometric rate experiments on iodobenzene sulphonation (nitromethane solution, $24^{\circ}-30^{\circ}$, wavelength $380 \mathrm{~m} \mu, 1 \mathrm{~cm}$ quartz-cuvettes).

\begin{tabular}{|c|c|c|c|c|c|}
\hline \multirow{2}{*}{$\begin{array}{l}\text { Expt. } \\
\text { No. }\end{array}$} & \multicolumn{2}{|c|}{ Initial concentration } & \multicolumn{2}{|c|}{ Max. absorbance } & \multirow{2}{*}{$\begin{array}{l}\text { Rate constant } \\
\text { sec. order } \\
\text { decay } \\
\text { absorb. }{ }^{-1} \times \text { sec }^{-1}\end{array}$} \\
\hline & $\begin{array}{c}\text { Iodobenzene } \\
\text { mole } / 1\end{array}$ & $\begin{array}{l}\text { Sulphurtrioxide } \\
\text { mole/l }\end{array}$ & $\begin{array}{l}\text { time } \\
\text { sec }\end{array}$ & $\begin{array}{l}\text { absorb- } \\
\text { ance }\end{array}$ & \\
\hline $\begin{array}{l}1 \\
2 \\
3\end{array}$ & $\begin{array}{c}1.30 \\
" \\
"\end{array}$ & $\begin{array}{c}0.50 \\
\Rightarrow \\
"\end{array}$ & $\begin{array}{l}2.1 \\
1.9 \\
3.8\end{array}$ & $\begin{array}{l}1.22 \\
1.46 \\
0.74\end{array}$ & $\begin{array}{l}0.23 \\
0.25 \\
0.23\end{array}$ \\
\hline $\begin{array}{l}4 \\
5 \\
6 \\
7 \\
8\end{array}$ & $\begin{array}{l}0.43 \\
0.63 \\
0.82 \\
1.18 \\
1.81 \\
\end{array}$ & $\begin{array}{l}0.36 \\
0.35 \\
0.35 \\
0.33 \\
0.30\end{array}$ & $\begin{array}{l}1.2 \\
2.1 \\
2.8 \\
1.2 \\
4.9\end{array}$ & $\begin{array}{l}0.32 \\
0.53 \\
0.72 \\
1.04 \\
1.30\end{array}$ & $\begin{array}{l}1.70 \\
0.88 \\
0.52 \\
0.27 \\
0.12\end{array}$ \\
\hline $\begin{array}{r}9 \\
10 \\
\end{array}$ & $\begin{array}{c}1.18 \\
"\end{array}$ & $\begin{array}{l}0.33 \\
0.61 \\
\end{array}$ & $\begin{array}{l}1.2 \\
2.1 \\
\end{array}$ & $\begin{array}{l}1.04 \\
1.61 \\
\end{array}$ & $\begin{array}{l}0.28 \\
0.31\end{array}$ \\
\hline $\begin{array}{l}11 \\
12 \\
13 \\
\end{array}$ & $\begin{array}{c}0.82 \\
" \\
" \\
\end{array}$ & $\begin{array}{c}0.33 \\
" \\
"\end{array}$ & $\begin{array}{l}3.0 \\
1.7 \\
3.0\end{array}$ & $\begin{array}{l}0.62 \\
0.90 \\
0.82 \\
\end{array}$ & $\begin{array}{l}0.35 \\
0.35 \\
0.30 \\
\end{array}$ \\
\hline $\begin{array}{l}14 \\
15\end{array}$ & $\begin{array}{c}0.82 \\
"\end{array}$ & $\begin{array}{l}0.27 \\
"+\mathrm{I}_{2}\end{array}$ & $\begin{array}{l}4.0 \\
2.8\end{array}$ & $\begin{array}{l}0.63 \\
1.38\end{array}$ & $\begin{array}{l}0.26 \\
0.23\end{array}$ \\
\hline
\end{tabular}

is the optical density in absorbance units at $t$ seconds, the second order law may be written

$$
1 / A-q=k^{\prime} \times t
$$

The slope of the graph determines the decay rate constant $k^{\prime}$. Second order constants and maximum absorbance at $24-30^{\circ}$ obtained at $380 \mathrm{~m} \mu$ have been compiled in Table 1 .

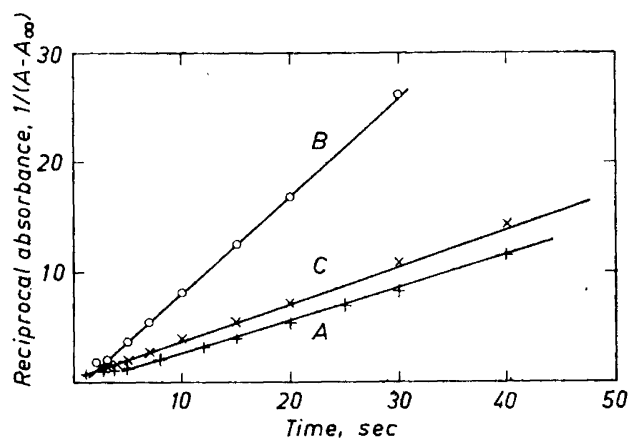

Fig. 3. Colour decay at $380 \mathrm{~m} \mu$ during the sulphonation of iodobenzene. A: $\left[\mathrm{SO}_{3}\right]_{0}=$ $0.33,\left[\mathrm{C}_{6} \mathrm{H}_{5} \mathrm{I}\right]_{0}=1.18 \mathrm{M}$. B: $\left[\mathrm{SO}_{3}\right]_{0}=0.35,\left[\mathrm{C}_{6} \mathrm{H}_{5} \mathrm{I}\right]_{0}=0.63 \mathrm{M}$. C: readings from curve $\mathrm{A}$, Fig. 2. $24-30^{\circ}$, nitromethane. $1 \mathrm{~cm}$ quartz cuvettes. 
Some idea of the reproducibility of data from shorttime experiments may be obtained from the series Nos. 1-3, in Table 1, where the parameter is the rate with which iodobenzene has been added. In Nos. 1 and 2 the iodobenzene has been added at a speed normally employed $(\sim 0.3 \mathrm{sec})$ and in No. 3 the operation was protracted to $3 \mathrm{sec}$. It will appear that the maximum absorbance, but not the rate of decomposition, is markedly influenced by the conditions of mixing.

The data of Nos. 4-8 demonstrate the marked influence of the iodobenzene (when in excess), on maximum absorbance as well as on decay rate. With good approximation the maximum absorbance is proportional to the initial concentration of iodobenzene, while the decay rate constant is inversely proportional to the iodobenzene concentration.

The sulphur trioxide concentration has little influence on the decay rate constant, $c f$. expts. Nos. $9-10$, but the max. optical density is approximately proportional to the initial sulphur trioxide concentration.

Two distillation fractions and a residue of iodobenzene, Nos. 11, 12 and 13 in Table 1, have been investigated under identical conditions. In spite of varying content of impurities, the final optical densities being $0.16,0.20$ and 0.89 , there is hardly any difference in the kinetic picture. Experiments Nos. 14 and 15 are identical, except that in the latter free iodine was present, $\left[\mathrm{I}_{2}\right]_{0}=0.0017 \mathrm{M}$. At $487 \mathrm{~m} \mu$ this quantity of iodine causes an absorption approximately equal to the maximum absorption at $380 \mathrm{~m} \mu$ observed in the same experiments. As will be seen, there is no influence of the free iodine upon the kinetics.

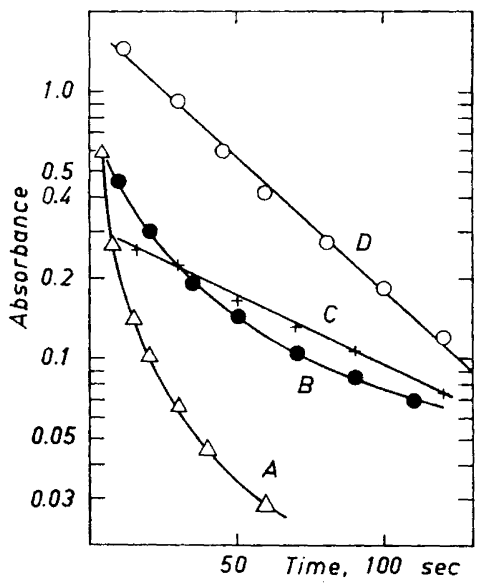

Fig. 4. Colour decay at $380 \mathrm{~m} \mu$ during the sulphonation of iodobenzene; the main parameter being sulphuric acid concentration. Concentration M:

$\begin{array}{cccc} & {\left[\mathrm{H}_{2} \mathrm{SO}_{4}\right]_{0}} & {\left[\mathrm{SO}_{3}\right]_{0}} & {\left[\mathrm{C}_{6} \mathrm{H}_{5} \mathrm{I}\right]_{0}} \\ \mathrm{~A} & 0 & 0.35 & 0.82 \\ \mathrm{~B} & 0.15 & 0.25 & 0.87 \\ \mathrm{C} & 0.49 & 0.19 & 0.94 \\ \mathrm{D} & 1.50 & 0.21 & 1.08\end{array}$

Acta Chem. Scand. 17 (1963) No. 8 
Some rate data were obtained for the short initial period at temperatures lower than room temperature, and it was evident that the formation rate at lower temperature was much lower than at room temperature, suggesting an appreciable activation energy for the formation of the coloured compound. $E . g$. in experiments with $\left[\mathrm{SO}_{3}\right]_{0}=0.18$ and $\left[\mathrm{C}_{6} \mathrm{H}_{5} \mathrm{I}\right]_{0}=1.18 \mathrm{M}$ the formation rate was 0.3 abs. units $/ \mathrm{sec}$ at $28^{\circ}$, but $0.06 \mathrm{abs}$. units $/ \mathrm{sec}$ at $13^{\circ}$.

Colour rate experiments in the presence of sulphuric acid. A curve representing one of these experiments is shown in Fig. 2 B. The effects of the sulphuric acid are to retard the formation of the colour, and to change the colour decay into a first order reaction.

Fig. 4 represents a series of experiments, in which the concentration of sulphuric acid is the main parameter. It will appear that a certain excess of sulphuric acid relative to sulphur trioxide is required to make the first order character evident.

Product rate experiments. Some runs are shown in Fig. 5. Initial product formation rates, as determined graphically from such curves, have been plotted versus product concentration at infinite time, $\left[\mathrm{RSO}_{3} \mathrm{H}\right]_{\infty}$, in Fig. 6 .

In spite of considerable experimental errors as well as errors in the determination of the "initial» rate from the curves, the following conclusion may be made:

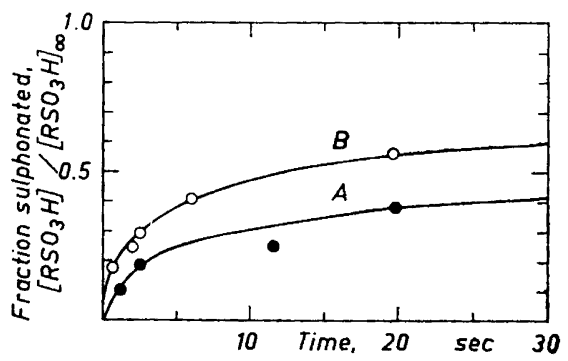

Fig. 5. Product formation rate data of sulphonation of iodobenzene in nitromethane. A: $0^{\circ}:\left[\mathrm{C}_{6} \mathrm{H}_{5} \mathrm{I}\right]_{0}=0.46,\left[\mathrm{RSO}_{3} \mathrm{H}\right]_{\infty}=0.11 \mathrm{M} . \mathrm{B}: 20^{\circ}:\left[\mathrm{C}_{6} \mathrm{H}_{5} \mathrm{I}\right]_{0}=1.18,\left[\mathrm{RSO}_{3} \mathrm{H}\right]_{\infty}=0.13 \mathrm{M}$.

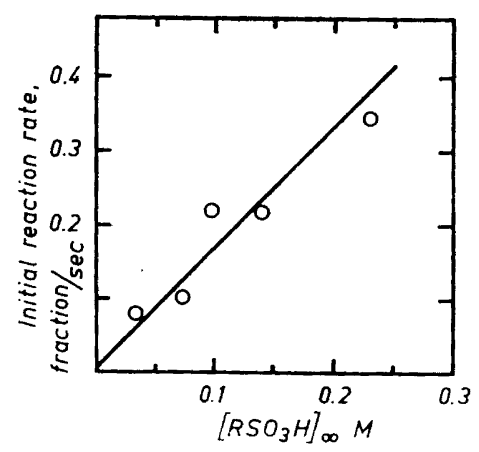

Fig. 6. Initial rate of product formation at $0^{\circ}$ at varying initial concentration of sulphur trioxide. Iodobenzene concentration $1.2 \mathrm{M}$. 
The vinitial» product formation rate is proportional to the square of the initial sulphur trioxide concentration. However, the reaction does not follow a simple second order scheme, the product formation being markedly retarded with time.

Closely similar results have previously been obtained by Hinshelwood et al. in an investigation on aromatic sulphonation. In these experiments nitrobenzene was used as solvent, various aromatics were sulphonated, among these chloro- and bromobenzene, but not iodobenzene..$^{4-5}$

\section{DISCUSSION}

The relatively fast formation of the coloured compound would lead to the immediate assumption that it is a complex of iodobenzene and sulphur trioxide.

A number of alternative explanations can be shown to be unlikely. None of the reaction products, sulphonic acid, sulphonic anhydride or sulphone form coloured complexes with sulphur trioxide or iodobenzene. It has been shown that free molecular iodine cannot be the colouring agent, and it appears unlikely that complexes of iodobenzene and iodine would give rise to absorption at $400 \mathrm{~m} \mu$, such complexes between aromatics and halogens usually showing absorption maximum in the neighbourhood of $300 \mathrm{~m} \mu .{ }^{15}$ Furthermore any such complex.would be expected to behave chemically like iodine, and it seems difficult to offer a mechanism, which could account for the observed rate laws.

Sulphone is formed as by-product in the sulphonation of iodobenzene, and the mechanism of sulphone formation in sulphonation reactions is not yet fully understood. It is likely (cf. Joly et $\left.a l .{ }^{16}\right)$ that the reaction is a sulphonylation according to the scheme:

$$
\begin{gathered}
\mathrm{RSO}_{3} \mathrm{H}+\mathrm{SO}_{3} \rightleftharpoons \mathrm{RSO}_{2}^{+}+\mathrm{HSO}_{4}^{-} \\
\mathrm{RSO}_{2}^{+}+\mathrm{RH} \rightarrow \mathrm{R}_{2} \mathrm{SO}_{2}+\mathrm{H}^{+}
\end{gathered}
$$

but no plausible intermediate occurring in such scheme would give rise to the observed kinetics of the colour decay.

It is therefore justifiable to consider the possibility of a complex between sulphur trioxide and iodobenzene in more detail.

For the development of rate expressions describing the colour-decay the following assumptions are made. The composition of the coloured complex, $\mathrm{C}_{1}$, is given by the formula: $\left(\mathrm{C}_{6} \mathrm{H}_{5} \mathrm{I}\right)_{\mathrm{n}}, \mathrm{SO}_{3}$. It is now assumed that the equilibrium

$$
\mathrm{n} \mathrm{RH}+\mathrm{SO}_{3} \rightleftharpoons \mathrm{C}_{1}
$$

is fast compared to the irreversible reaction causing the disappearance of the colour. Furthermore it will be assumed that the concentrations of all complexes of iodobenzene and sulphur trioxide (including such as may occur in the sulphonation mechanism) can be neglected.

Since iodobenzene has been in excess in these experiments the concentration of iodobenzene may be regarded as constant during a run. It is therefore readily seen that the concentration of $\mathrm{C}_{1}$ at any time (apart from the first seconds where equilibrium (3) has not been attained) is proportional to the sulphur trioxide concentration, so that the colour decay curve represents the disappearance of the sulphur trioxide. 
The observation that the colour decay follows a second order scheme in the aprotic mixtures, but a first order scheme, when sulphuric acid is present, is consistent with this hypothesis, as will appear from the following.

For aprotic sulphonation the product formation has previously been shown ${ }^{3}$ to follow the expression:

$$
\mathrm{d}\left[\mathrm{RSO}_{3} \mathrm{H}\right] / \mathrm{d} t=k_{\mathrm{a}} \cdot[\mathrm{RH}] \cdot\left[\mathrm{SO}_{3}\right]^{2}
$$

and the rate-measurements on product formation in the present system confirm this expression.

For sulphonation in protic systems it has been shown ${ }^{9,11}$ that the rate of product formation follows the expression:

$$
\mathrm{d}\left[\mathrm{RSO}_{3} \mathrm{H}\right] / \mathrm{dt}=k_{\mathrm{p}} \cdot[\mathrm{RH}] \cdot\left[\mathrm{SO}_{3}\right] \cdot\left[\mathrm{H}^{+}\right]
$$

Since all other concentrations than those of reactants and products are assumed negligible, the following equation may be written:

$$
-\mathrm{d}\left[\mathrm{SO}_{3}\right] / \mathrm{dt}=\mathrm{d}\left[\mathrm{RSO}_{3} \mathrm{H}\right] / \mathrm{dt}
$$

and it is now readily seen that the disappearance of the sulphur trioxide will follow a second order scheme in aprotic medium, but a first order scheme in protic medium.

As regards the slow formation of the colour in the experiments with sulphuric acid, this may be associated with the equilibrium:

$$
\mathrm{H}_{2} \mathrm{SO}_{4}+\mathrm{SO}_{3} \rightleftharpoons \mathrm{H}_{2} \mathrm{~S}_{2} \mathrm{O}_{7}
$$

By introducing the mass-law for eqn. (3) into both sides of eqn. (6) the following expression is derived:

$$
-\mathrm{d}\left[\mathrm{C}_{1}\right] / \mathrm{d} t=k^{\prime \prime} \cdot\left[\mathrm{C}_{1}\right]^{2} /[\mathrm{RH}]^{\mathrm{n}-1}
$$

Since the rate constant for the colour decay was observed to be approximately proportional to the reciproque value of the iodobenzene concentration, $c f$. Table $\mathrm{I}$, the rate data are consistent with eqn. (8) provided $\mathrm{n}=2$, i.e. the composition of the complex should be $\left(\mathrm{C}_{6} \mathrm{H}_{5} \mathrm{I}\right)_{2}, \mathrm{SO}_{3}$.

It would not be advisable at the present stage to consider the structure of the complex in more detail; it does, however, not appear likely that this complex represents an activated intermediate stage in the sulphonation reaction.

The assumptions made above, that eqn. (3) is fast and that complex concentrations are negligible, are partly justified by similar assumptions often being made when considering electrophilic substitutions. However, they may not be applicable to the present system. A possibility, which deserves special attention, is that the coloured complex is the $1: 1$ complex $\left(\mathrm{C}_{2}\right.$, below) often stated to be an intermediate in the sulphonation. In the aprotic system the mechanism would be:

$$
\begin{aligned}
& \mathrm{RH}+\mathrm{SO}_{3} \underset{k_{-1}}{\stackrel{k_{1}}{\rightleftharpoons}} \mathrm{R} \overbrace{\mathrm{SO}_{3}}^{\mathrm{H}} \\
& \mathrm{C}_{2}+\mathrm{SO}_{3} \stackrel{k_{2}}{\longrightarrow} \mathrm{RSO}_{3} \mathrm{SO}_{3} \mathrm{H}\left(\stackrel{\left(\mathrm{C}_{2}\right)}{\left.\rightleftharpoons \mathrm{RSO}_{3} \mathrm{H}+\mathrm{SO}_{3}\right)}\right.
\end{aligned}
$$

Acta Chem. Scand. 17 (1963) No. 8 
For the disappearance of $\mathrm{C}_{2}$ the following rate expression can be derived:

$$
-\mathrm{d}\left[\mathrm{C}_{2}\right] / \mathrm{d} t=\frac{2 \cdot k_{1} \cdot k_{2}\left[\mathrm{C}_{2}\right]^{2}}{k_{1}[\mathrm{RH}]+k_{-1}}
$$

Consistency with the observed kinetics of the colour decay is obtained by assuming $k_{1}[\mathrm{RH}]>k_{-1}$. This condition implies, however, that the sulphur trioxide concentration is negligible, so that

$$
\left[\mathrm{C}_{2}\right] \cong\left[\mathrm{SO}_{3}\right]_{0}-\left[\mathrm{RSO}_{3} \mathrm{H}\right]
$$

where index 0 stands for zero time.

By inserting (12) into (11) a rate expression is developed for product formation. This expression contains the concentration of the aromatic in the denominator, in contrast to eqn. (4), and this explanation is therefore inapplicable.

The fact that temporary light absorbance also has been observed in the sulphonation of chloro- and bromobenzene would indicate that sulphur trioxide forms complexes with many aromatics. The absorption within the visible range, observed in the iodobenzene sulphur trioxide complex, may be attributed to the presence of the carbon-iodine bond. Other compounds containing the carbon-iodine bond have been recorded to exhibit somewhat peculiar spectroscopic characteristics. ${ }^{17}$

Acknowledgement. The author wishes to express his gratitude to Dr. C. F. Jacobsen, C.U. Linderstrøm-Lang and Th. Rosenberg for helpful discussions throughout the course of this work. The author is also indebted to Mr. Elfin Larsen for the gas-chromatographic data.

\section{REFERENCES}

1. Ingold, C. K. Structure and Mechanism in Organic Chemistry, Cornell Univ. Press, New York 1953, pp. $299-302$.

2. Brown, H. C. and Nelson, K. L. R. The Chemistry of Petroleum Hydrocarbons, Reinhold Publishing Co., New York 1955, Vol 3. pp. 465-578.

3. Vicary, D. R. and Hinshelwood, C. N. J. Chem. Soc. 19391372.

4. Wadsworth, K. D. and Hinshelwood, C. N. J. Chem. Soc. 1944469.

5. Dresel, E. and Hinshelwood, C. N. J. Chem. Soc. 1944649.

6. Gilbert, E. E. Chem. Rev. 62 (1962) 549.

7. Melander, L. Arkiv Kemi 2 (1950) 211.

8. Berglund-Larsson, U. and Melander, L. Arkiv Kemi 6 (1954) 219.

9. Brand, J. C. D., Jarvie, A. W. P. and Horning, W. C. J. Chem. Soc. 19593844.

10. Gold, V. and Satchell, D. P. N. J. Chem. Soc. 19561635.

11. Davenport, D. A. and Walsh, J. A. Abstr. Paper 134th Meeting Am. Chem. Soc. 1958, $30 \mathrm{P}$.

12. Christensen, N. H. Acta Chem. Scand. 15 (1961) 1507.

13. Terentev, A. P., Yanovskaya, L. A., Berlin, A. M. and Borisov, E. A. Vestn. Mosk. Univ., Ser. Fiz.-Mat. 4 (1953) No. 6, 117.

14. Ratcliff, G. A. Kinetics of Sulphonation. Univ. Microfilm Publ. No. 9798. Ann. Arbor, Michigan 1954.

15. Keefer, R. M. and Andrews, L. J. J. Am. Chem. Soc. 72 (1950) 5170.

16. Joly, R., Bucourt, R. and Mathieu, J. Rec. Trav. Chim. 78 (1959) 527.

17. Durie, R. A., Iredale, T. and Jarvie, L. M. S. J. Chem. Soc. 19501181.

Received May 27, 1963.

Acta Chem. Scand. 17 (1963) No. 8 